\title{
Fredholm spectrum and growth of cohomology groups
}

\author{
by
}

\author{
JÖRG EschMEIER (Saarbrücken)
}

\begin{abstract}
Let $T \in L(E)^{n}$ be a commuting tuple of bounded linear operators on a complex Banach space $E$ and let $\sigma_{\mathrm{F}}(T)=\sigma(T) \backslash \sigma_{\mathrm{e}}(T)$ be the non-essential spectrum of $T$. We show that, for each connected component $M$ of the manifold $\operatorname{Reg}\left(\sigma_{\mathrm{F}}(T)\right)$ of all smooth points of $\sigma_{\mathrm{F}}(T)$, there is a number $p \in\{0, \ldots, n\}$ such that, for each point $z \in M$, the dimensions of the cohomology groups $H^{p}\left((z-T)^{k}, E\right)$ grow at least like the sequence $\left(k^{d}\right)_{k \geq 1}$ with $d=\operatorname{dim} M$.
\end{abstract}

0. Introduction. Let $T=\left(T_{1}, \ldots, T_{n}\right) \in L(E)^{n}$ be a commuting tuple of bounded linear operators on a complex Banach space $E$. The joint spectrum $\sigma(T)$ of $T$ in the sense of J. L. Taylor is the set of all points $z \in \mathbb{C}^{n}$ for which the Koszul complex $K^{\bullet}(z-T, E)$ of the commuting tuple $z-T=\left(z_{1}-T_{1}, \ldots, z_{n}-T_{n}\right)$ is not exact. By definition the Koszul complex $K^{\bullet}(T, E)$ is a finite complex of Banach spaces

$$
0 \rightarrow \Lambda^{0}(s, E) \stackrel{T}{\rightarrow} \Lambda^{1}(s, E) \stackrel{T}{\rightarrow} \cdots \stackrel{T}{\rightarrow} \Lambda^{n}(s, E) \rightarrow 0,
$$

where $\Lambda^{p}(s, E)$ consists of all forms of degree $p$ in $n$ indeterminates $s=$ $\left(s_{1}, \ldots, s_{n}\right)$ with coefficients in $E$ and the coboundary maps act as

$$
T \sum_{1 \leq i_{1}<\cdots<i_{p} \leq n} x_{i_{1} \ldots i_{p}} s_{i_{1}} \wedge \cdots \wedge s_{i_{p}}=\sum_{j=1}^{n} \sum_{1 \leq i_{1}<\cdots<i_{p} \leq n} T_{j} x_{i_{1} \ldots i_{p}} s_{j} \wedge s_{i_{1}} \wedge \cdots \wedge s_{i_{p}} .
$$

The Taylor spectrum $\sigma(T)$ of $T$ is a compact, non-empty subset of $\mathbb{C}^{n}$ which carries a natural multidimensional analytic functional calculus [8].

The commuting tuple $T$ is said to be Fredholm if all cohomology groups $H^{p}(T, X)(p=0, \ldots, n)$ of its Koszul complex $K^{\bullet}(T, E)$ are finite-dimen-

2000 Mathematics Subject Classification: Primary 47A13; Secondary 47A53, 13D40, $32 \mathrm{C} 35$.

Key words and phrases: Fredholm spectrum, cohomology groups, Hilbert-Samuel polynomial. 
sional. In this case the index of $T$ is defined as the Euler characteristic

$$
\operatorname{ind}(T)=\sum_{p=0}^{n}(-1)^{p} \operatorname{dim} H^{p}(T, X)
$$

of its Koszul complex. The essential spectrum $\sigma_{\mathrm{e}}(T)$ of $T$ consists of all points $z \in \mathbb{C}^{n}$ for which $z-T$ is not Fredholm and the essential resolvent set is the complement $\varrho_{\mathrm{e}}(T)=\mathbb{C}^{n} \backslash \sigma_{\mathrm{e}}(T)$ of the essential spectrum.

The observation that a commuting tuple $T$ is Fredholm if and only if the cohomology sheaves $H^{p}\left(z-T, \mathcal{O}_{\mathbb{C}^{n}}^{E}\right)(p=0, \ldots, n)$ of the associated complex $K^{\bullet}\left(z-T, \mathcal{O}_{\mathbb{C}^{n}}^{E}\right)$ of Banach-free analytic sheaves are coherent near $0 \in \mathbb{C}^{n}$ implies that the inessential spectrum or Fredholm spectrum of $T$

$$
\sigma_{\mathrm{F}}(T)=\sigma(T) \backslash \sigma_{\mathrm{e}}(T)=\bigcup_{p=0}^{n} \operatorname{supp}\left(\mathcal{H}^{p}\right),
$$

where $\mathcal{H}^{p}=H^{p}\left(z-T, \mathcal{O}_{\varrho_{\mathrm{e}}(T)}^{E}\right)$, is an analytic subset of $\varrho_{\mathrm{e}}(T)$ (Chapter 10 in $[5])$. Hence the smooth points of $\sigma_{\mathrm{F}}(T)$ form a dense open subset $\operatorname{Reg}\left(\sigma_{\mathrm{F}}(T)\right)$ which is a submanifold of $\mathbb{C}^{n}$.

Let $C$ be a connected component of $\varrho_{\mathrm{e}}(T)$. For $p \in\{0, \ldots, n\}$, define $c_{p}=\min \left\{\operatorname{dim} H^{p}(z-T, E) ; z \in C\right\}$. Then the discontinuity points of the functions

$$
C \rightarrow \mathbb{N}, \quad z \mapsto \operatorname{dim} H^{p}(z-T, E) \quad(p \in\{0, \ldots, n\})
$$

are proper analytic subsets of $C$ given by $\left\{z \in C\right.$; $\left.\operatorname{dim} H^{p}(z-T, E)>c_{p}\right\}$. Using the fact that $c_{p}$ is the rank of the coherent sheaf $\mathcal{H}^{p}$ on $C$, and hence can be computed as the Samuel multiplicity of its stalks, it was shown in [4] that

$$
\lim _{k \rightarrow \infty} \frac{\operatorname{dim} H^{p}\left((z-T)^{k}, E\right)}{k^{n}}=c_{p} \quad(p \in\{0, \ldots, n\})
$$

for each point $z \in C$. If $c_{p}>0$, then $C$ is a connected component of the manifold $\operatorname{Reg}\left(\sigma_{\mathrm{F}}(T)\right)$ of dimension $n$ and the sequence $\left(\operatorname{dim} H^{p}\left((z-T)^{k}, E\right)\right)_{k \geq 1}$ grows precisely like the sequence $\left(k^{n}\right)_{k \geq 1}$ for each $z \in C$. If $c_{p}=0$, then the above limit formula only gives an upper estimate. It is the aim of the present note to show that, for every connected component $M$ of $\operatorname{Reg}\left(\sigma_{\mathrm{F}}(T)\right)$ of dimension $d$, there is a number $p \in\{0, \ldots, n\}$ such that the sequence $\left(\operatorname{dim} H^{p}\left((z-T)^{k}, E\right)_{k \geq 1}\right.$ grows at least like the sequence $\left(k^{d}\right)_{k \geq 1}$ for every $z \in M$.

Particular cases of this result, together with the Scott Brown technique, have been used to obtain joint invariant subspace results for commuting tuples of contractions (see [2] and [1]). The question whether also a corresponding upper estimate holds remains open. 
1. Coherent sheaves. Let $\mathcal{F}$ be a coherent sheaf on a complex manifold $X$. The section spaces $\mathcal{F}(U), U \subset X$ open, carry a canonical nuclear Fréchetspace topology (see Section 4.1 in [5]). For a Banach space $E$, we denote by $\mathcal{F}^{E}=\mathcal{F} \widehat{\otimes} E$ the sheaf of $\mathcal{O}_{X}$-modules given by the presheaf

$$
U \mapsto \mathcal{F}(U) \widehat{\otimes} E \quad(U \subset X \text { open })
$$

Here $\widehat{\otimes}$ denotes the projective (or injective) tensor product of locally convex spaces. Since this presheaf satisfies the sheaf axioms, there are natural identifications $\mathcal{F}^{E}(U) \cong \mathcal{F}(U) \widehat{\otimes} E$ for each open set $U \subset X$.

For each sequence $\mathcal{F} \rightarrow \mathcal{G} \rightarrow \mathcal{H}$ of coherent $\mathcal{O}_{X}$-sheaves, the resulting sequences of section spaces

$$
\mathcal{F}(U) \widehat{\otimes} E \rightarrow \mathcal{G}(U) \widehat{\otimes} E \rightarrow \mathcal{H}(U) \widehat{\otimes} E
$$

remain exact on all Stein open subsets $U \subset X$. Therefore the functor of passing from coherent analytic sheaves $\mathcal{F}$ over $X$ to the stalks $\mathcal{F}_{x}^{E}$ of the tensorized sheaf $\mathcal{F}^{E}=\mathcal{F} \widehat{\otimes} E$ at a given point $x \in X$ is exact.

Suppose, in addition, that $X \subset \mathbb{C}^{n}$ is a complex submanifold. Let $T=$ $\left(T_{1}, \ldots, T_{n}\right) \in L(E)^{n}$ be a commuting tuple of bounded operators on a complex Banach space $E$, and let $\mathcal{F}$ be a coherent sheaf on $X$. Then, for every open set $U \subset X$, we may regard the tuple $z-T=\left(z_{1}-T_{1}, \ldots, z_{n}-T_{n}\right)$ as a commuting tuple of continuous linear operators on the Fréchet space $\mathcal{F}^{E}(U)=\mathcal{F}(U) \widehat{\otimes} E$. We denote by $K^{\bullet}\left(z-T, \mathcal{F}^{E}(U)\right)$ the Koszul complex of this commuting tuple and write as usual

$$
H^{j}\left(z-T, \mathcal{F}^{E}(U)\right) \quad(j=0, \ldots, n)
$$

for the cohomology spaces of the Koszul complex $K^{\bullet}\left(z-T, \mathcal{F}^{E}(U)\right)$. Similar notations will be used for the induced sequences of sheaves $K^{\bullet}\left(z-T, \mathcal{F}^{E}\right)$ or stalks $K^{\bullet}\left(z-T, \mathcal{F}_{x}^{E}\right)$ at a given point $x \in X$.

Proposition 1.1. Let $X \subset \mathbb{C}^{n}$ be a complex submanifold with $0 \in X$, and let $T \in L(E)^{n}$ be a commuting tuple of bounded operators on a complex Banach space E. Suppose that $p \in\{0, \ldots, n\}$ is an integer with

$$
H^{j}\left(z-T, \mathcal{O}_{X, 0}^{E}\right)=0 \quad(j=p+1, \ldots, n) .
$$

Then, for each epimorphism $\mathcal{G} \stackrel{q}{\rightarrow} \mathcal{H}$ of coherent $\mathcal{O}_{X}$-sheaves $\mathcal{G}$ and $\mathcal{H}$, the induced map

$$
H^{p}\left(z-T, \mathcal{G}_{0}^{E}\right) \stackrel{q}{\rightarrow} H^{p}\left(z-T, \mathcal{H}_{0}^{E}\right)
$$

is surjective.

Proof. Since $\mathcal{G}$ and $\mathcal{H}$ are coherent $\mathcal{O}_{X}$-sheaves, the same is true for the kernel sheaf $\mathcal{F}=\operatorname{Ker}(q: \mathcal{G} \rightarrow \mathcal{H})$. By shrinking $X$ to a sufficiently small open neighbourhood of 0 in $X$, we may suppose that $\mathcal{F}$ has a finite free resolution of the form

$$
0 \rightarrow \mathcal{O}_{X}^{r_{q}} \rightarrow \mathcal{O}_{X}^{r_{q-1}} \rightarrow \cdots \rightarrow \mathcal{O}_{X}^{r_{0}} \rightarrow \mathcal{F} \rightarrow 0
$$


The remarks preceding the proposition show that the induced sequences

$$
0 \rightarrow \mathcal{F}_{0}^{E} \rightarrow \mathcal{G}_{0}^{E} \stackrel{q}{\rightarrow} \mathcal{H}_{0}^{E} \rightarrow 0
$$

and

$$
0 \rightarrow \mathcal{O}_{X, 0}^{E^{r_{q}}} \rightarrow \mathcal{O}_{X, 0}^{E^{r_{q-1}}} \rightarrow \cdots \rightarrow \mathcal{O}_{X, 0}^{E^{r_{0}}} \rightarrow \mathcal{F}_{0}^{E} \rightarrow 0
$$

remain exact. Let us consider the double complex

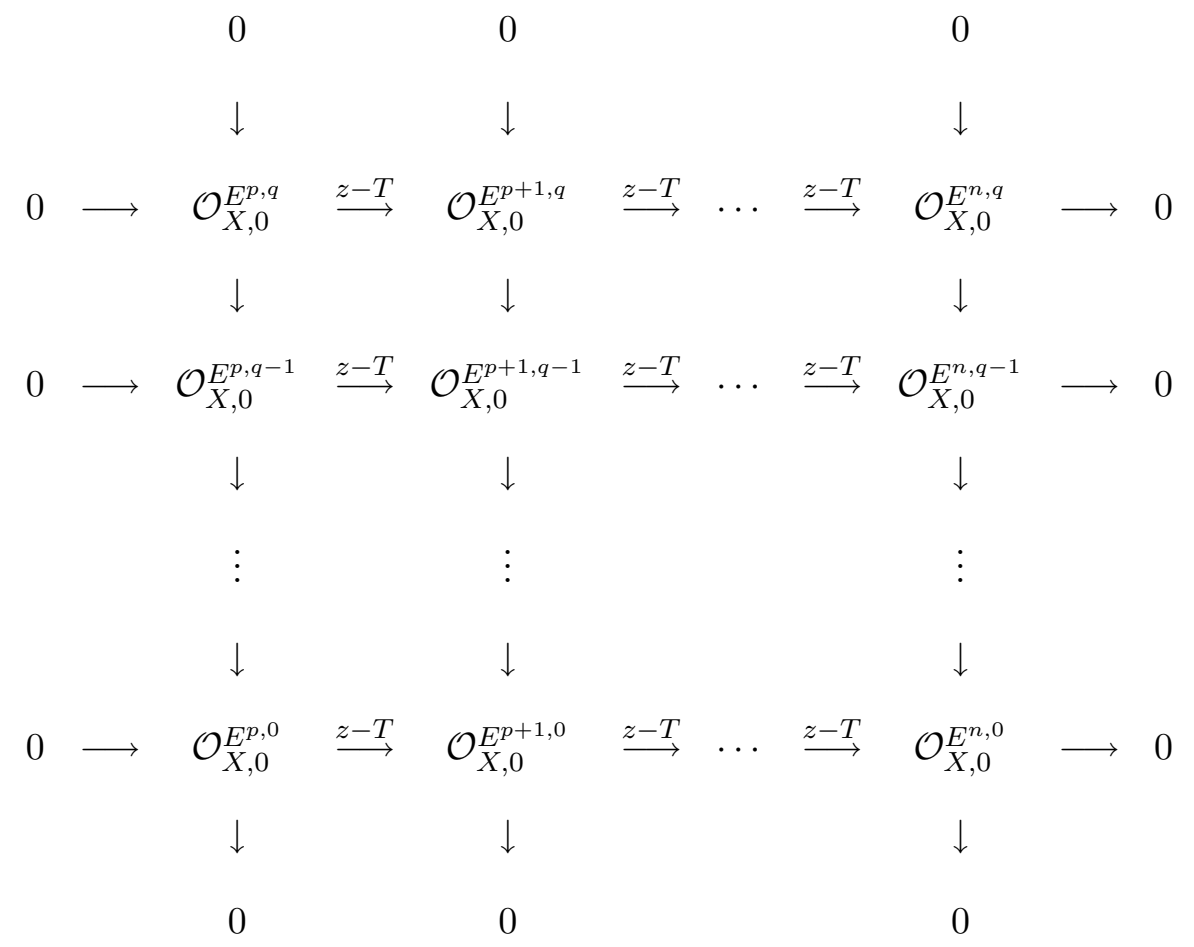

where $E^{i, j}=\Lambda^{i}(s, E)^{r_{j}}$, the rows are given by (direct sums of) the Koszul complex $K^{\bullet}\left(z-T, \mathcal{O}_{X, 0}^{E}\right)$ and the columns are obtained from the free resolution of the kernel sheaf $\mathcal{F}$ chosen above. By construction all rows of this double complex are exact except in the first place, and all columns are exact except in the last place.

Standard double complex methods imply that $H^{j}\left(z-T, \mathcal{F}_{0}^{E}\right)=0$ for $j=p+1, \ldots, n$ (Lemma A2.6 in [5]). Hence the long exact cohomology sequence induced by the short exact sequence of Koszul complexes

$$
0 \rightarrow K^{\bullet}\left(z-T, \mathcal{F}_{0}^{E}\right) \rightarrow K^{\bullet}\left(z-T, \mathcal{G}_{0}^{E}\right) \rightarrow K^{\bullet}\left(z-T, \mathcal{H}_{0}^{E}\right) \rightarrow 0
$$


is of the form

$$
\begin{aligned}
& 0 \rightarrow H^{0}\left(z-T, \mathcal{F}_{0}^{E}\right) \rightarrow H^{0}\left(z-T, \mathcal{G}_{0}^{E}\right) \rightarrow H^{0}\left(z-T, \mathcal{H}_{0}^{E}\right) \\
& \rightarrow H^{1}\left(z-T, \mathcal{F}_{0}^{E}\right) \rightarrow \ldots \\
& \rightarrow H^{p}\left(z-T, \mathcal{F}_{0}^{E}\right) \rightarrow H^{p}\left(z-T, \mathcal{G}_{0}^{E}\right) \rightarrow H^{p}\left(z-T, \mathcal{H}_{0}^{E}\right) \rightarrow 0 .
\end{aligned}
$$

In particular the last map is surjective.

Let $\Omega \subset \mathbb{C}^{n}$ be an open set with $0 \in \Omega$, and let $\mathcal{H}$ be a coherent sheaf on $\Omega$. Fix an arbitrary connected open zero neighbourhood $U \subset \Omega$. The set $A$ of all points $z \in U$ for which $\mathcal{H}$ is not locally free at $z$ is a nowhere dense analytic subset of $U$ and its complement $U \backslash A$ in $U$ is connected ([7, pp. 92 and 145]). The rank $\mathrm{rk}_{U} \mathcal{H}$ is by definition the constant value of the function $\operatorname{rk}_{\mathcal{O}_{z}}\left(\mathcal{H}_{z}\right)$ (the rank of the $\mathcal{O}_{z}$-module $\mathcal{H}_{z}$ ) for $z \in U \backslash A$. Since this number does not depend on the choice of $U$, one may define the rank of $\mathcal{H}$ at 0 as $\mathrm{rk}_{0} \mathcal{H}=\mathrm{rk}_{U} \mathcal{H}$.

Let $M^{\bullet}=\left(M^{p}, u^{p}\right)_{p=0}^{n}$ be a finite analytically parametrized complex of Banach spaces $M^{p}$ over $\Omega$ such that $\operatorname{dim} H^{p}\left(d^{\bullet}(0), M^{\bullet}\right)<\infty$ for $p=$ $0, \ldots, n$. It is well known (Proposition 9.4.5 and Remark 9.4.6 in [5]) that there exist an analytically parametrized complex $L^{\bullet}=\left(L^{p}, u^{p}\right)_{p=0}^{n}$ of finitedimensional vector spaces $L^{p}$ on a suitable open zero neighbourhood $U \subset \Omega$ and a family $h=\left(h^{p}\right)_{p=0}^{n}$ of holomorphic mappings $h^{p} \in \mathcal{O}\left(U, L\left(L^{p}, M^{p}\right)\right)$ such that the resulting maps

$$
\left(L^{\bullet}, u^{\bullet}(z)\right) \stackrel{h^{\bullet}(z)}{\longrightarrow}\left(M^{\bullet}, d^{\bullet}(z)\right) \quad(z \in U)
$$

as well as the induced maps between the associated complexes of sheaves

$$
\left(\mathcal{O}_{U}^{L^{\bullet}}, u^{\bullet}\right) \stackrel{h^{\bullet}}{\longrightarrow}\left(\mathcal{O}_{U}^{M^{\bullet}}, d^{\bullet}\right)
$$

are quasi-isomorphisms. Let us restrict to the case where $U$ is connected. Then, by Corollary 9.4.7 in [5], the discontinuity sets of the functions

$$
U \rightarrow \mathbb{N}, \quad z \mapsto \operatorname{dim} H^{p}\left(d^{\bullet}(z), M^{\bullet}\right)=\operatorname{dim} H^{p}\left(u^{\bullet}(z), L^{\bullet}\right)
$$

$(p=0, \ldots, n)$ are given by the proper analytic subsets

$$
S_{p}=\left\{z \in U ; \operatorname{dim} H^{p}\left(d^{\bullet}(z), M^{\bullet}\right)>c_{p}\right\} \subset U,
$$

where $c_{p}=\min \left\{\operatorname{dim} H^{p}\left(d^{\bullet}(z), M^{\bullet}\right) ; z \in U\right\}$.

Lemma 1.2. Let $M^{\bullet}=\left(M^{p}, d^{p}\right)_{p=0}^{n}$ be a finite analytically parametrized complex of Banach spaces on an open set $\Omega \subset \mathbb{C}^{n}$ containing $0 \in \mathbb{C}^{n}$ such that $\operatorname{dim} H^{p}\left(d^{\bullet}(z), M^{\bullet}\right)<\infty$ for $p=0, \ldots, n$ and $z \in \Omega$. Let $U \subset \Omega$ be an arbitrary connected open neighbourhood of $0 \in \mathbb{C}^{n}$. Then the cohomology 
sheaves $\mathcal{H}^{p}=H^{p}\left(d^{\bullet}, \mathcal{O}_{\Omega}^{M^{\bullet}}\right)(p=0, \ldots, n)$ are coherent and, for $p=0, \ldots, n$, we have

$$
\operatorname{rk}_{0}\left(\mathcal{H}^{p}\right)=\min \left\{\operatorname{dim} H^{p}\left(d^{\bullet}(z), M^{\bullet}\right) ; z \in U\right\} .
$$

Furthermore, the sets

$$
S_{p}=\left\{z \in U ; \operatorname{dim} H^{p}\left(d^{\bullet}(z), M^{\bullet}\right)>\operatorname{rk}_{0}\left(\mathcal{H}^{p}\right)\right\} \subset U
$$

are proper analytic subsets for $p=0, \ldots, n$.

Proof. The coherence of the cohomology sheaves $\mathcal{H}^{p}$ is well known and follows directly from the fact that the complex $M^{\bullet}=\left(M^{p}, d^{p}\right)_{p=0}^{n}$ is locally quasi-isomorphic to a complex $L^{\bullet}=\left(L^{p}, u^{p}\right)_{p=0}^{n}$ of finite-dimensional vector spaces.

By Corollary 9.4.7 in [5] the minimum occurring in the lemma is the constant value of the function $z \mapsto \operatorname{dim} H^{p}\left(d^{\bullet}(z), M^{\bullet}\right)$ outside a proper analytic subset of $U$. Since this number does not depend on the choice of $U$, we may suppose that there is an analytically parametrized complex $L^{\bullet}=\left(L^{p}, u^{p}\right)_{p=0}^{n}$ of finite-dimensional vector spaces on $U$ which is quasi-isomorphic to $M^{\bullet}$ in the sense explained before the lemma.

Let $A_{p}$ be the set of points $z \in U$ for which $\mathcal{H}^{p}$ is not locally free at $z$ and let $\mu \in U \backslash A_{p}$ be arbitrary. By an inductive application of Lemma 9.4.4 in [5], starting on the right-hand side of the complex

$$
0 \rightarrow L^{0} \stackrel{u^{0}}{\longrightarrow} L^{1} \stackrel{u^{1}}{\longrightarrow} \cdots \stackrel{u^{n-1}}{\longrightarrow} L^{n} \rightarrow 0,
$$

one can show that there is an open neighbourhood $W$ of $\mu$ in $U$ such that all sheaves $\operatorname{Ker}\left(u^{p}: \mathcal{O}_{W}^{L^{p}} \rightarrow \mathcal{O}_{W}^{L^{p+1}}\right)$ and $\operatorname{Im}\left(u^{p}: \mathcal{O}_{W}^{L^{p}} \rightarrow \mathcal{O}_{W}^{L^{p+1}}\right.$ ) are (up to isomorphisms) free $\mathcal{O}_{W}$-sheaves and such that there are vector-space isomorphisms $H^{p}\left(u^{\bullet}(z), L^{\bullet}\right) \cong \mathcal{H}_{z}^{p} \otimes_{\mathcal{O}_{z}}\left(\mathcal{O}_{z} / m_{z}\right)$ for $p=0, \ldots, n$ and $z \in W$. Here $m_{z}$ denotes the maximal ideal of the local ring $\mathcal{O}_{z}$. Since the $\mathbb{C}$-linear dimension of the module tensor product on the right is the rank of $\mathcal{H}_{z}^{p}$ as an $\mathcal{O}_{z}$-module, this observation completes the proof.

In the next section we apply Lemma 1.2 to analytically parametrized complexes arising as Koszul complexes of commuting multioperators.

2. Fredholm theory. Let $T=\left(T_{1}, \ldots, T_{n}\right) \in L(E)^{n}$ be a Fredholm tuple on a complex Banach space $E$. Suppose that $p \in\{0, \ldots, n\}$ is an integer with $H^{p}(T, E) \neq 0$ and

$$
H^{j}(T, E)=0 \quad(j=p+1, \ldots, n) .
$$

By Lemma 2.1.5 in [5] it follows that

$$
H^{j}\left(z-T, \mathcal{O}_{\mathbb{C}^{n}, 0}^{E}\right)=0 \quad(j=p+1, \ldots, n) .
$$

Corollary 9.4.7 in [5] implies that

$$
A=\left\{z \in \varrho_{\mathrm{e}}(T) ; H^{p}(z-T, E) \neq 0\right\} \subset \varrho_{\mathrm{e}}(T)
$$


is an analytic subset. Let us assume in the following that $\operatorname{dim}_{0}(A)>0$ and that 0 is a regular point of $A$, that is, there is a biholomorphic map $f: X \rightarrow Y$ from a suitable open neighbourhood $X$ of 0 in $A$ onto an open connected zero neighbourhood $Y \subset \mathbb{C}^{d}$ such that $f(0)=0$. Fix an open neighbourhood $U$ of 0 in $\mathbb{C}^{n}$ with $X=A \cap U$. By construction the minimum

$$
c=\min \left\{\operatorname{dim} H^{p}(z-T, E) ; z \in X\right\}
$$

is a positive integer. It follows from Corollary 9.4.7 in [5] that the discontinuity set of the function

$$
X \rightarrow \mathbb{N}, \quad z \mapsto \operatorname{dim} H^{p}(z-T, E),
$$

is given by the proper analytic subset

$$
S=\left\{z \in X ; \operatorname{dim} H^{p}(z-T, E)>c\right\} \subset X .
$$

Via the biholomorphic map $f: X \rightarrow Y$ the set $S$ corresponds to a proper analytic subset $\widetilde{S}=f(S) \subset Y$ such that

$$
\operatorname{dim} H^{p}\left(f^{-1}(z)-T, E\right)>c=\operatorname{dim} H^{p}\left(f^{-1}(w)-T, E\right)
$$

for all $z \in \widetilde{S}$ and $w \in Y \backslash \widetilde{S}$. Using the fact that proper analytic subsets of connected open sets in $\mathbb{C}^{d}$ have $2 d$-dimensional Lebesgue measure zero, one easily sees that the number $c$ does not depend on the choice of $X$.

Replacing $Y$ (and $X$ ) by smaller zero neighbourhoods, we may suppose that there exist an analytically parametrized complex $L^{\bullet}=\left(L^{j}, u^{j}\right)_{j=0}^{n}$ of finite-dimensional vector spaces on $Y$ and a family of holomorphic mappings

$$
h^{j} \in \mathcal{O}\left(Y, L\left(L^{j}, \Lambda^{j}(s, E)\right)\right)
$$

such that the resulting maps

$$
\left(L^{\bullet}, u^{\bullet}(z)\right) \stackrel{h^{\bullet}(z)}{\longrightarrow} K^{\bullet}\left(f^{-1}(z)-T, E\right) \quad(z \in Y)
$$

as well as the induced cochain maps between the associated complexes of sheaves

$$
\mathcal{O}_{Y}^{L^{\bullet}} \stackrel{h^{\bullet}}{\longrightarrow} K^{\bullet}\left(f^{-1}-T, \mathcal{O}_{Y}^{E}\right)
$$

are quasi-isomorphisms (Proposition 9.4.5 and Remark 9.4.6 in [5]). In particular, all cohomology sheaves $\mathcal{H}^{j}=H^{j}\left(f^{-1}-T, \mathcal{O}_{Y}^{E}\right)(j=0, \ldots, n)$ are coherent $\mathcal{O}_{Y}$-sheaves. By Lemma 1.2 we know that $c=\operatorname{rk}_{0}\left(\mathcal{H}^{p}\right)$.

On the other hand, it is well-known (see [6] or [4]) that the rank of the coherent sheaf $\mathcal{H}^{p}$ at $z=0$ is given by the Samuel multiplicity of its stalk $\mathcal{H}_{0}^{p}$ at $z=0$, that is,

$$
\operatorname{rk}_{0}\left(\mathcal{H}^{p}\right)=d ! \lim _{k \rightarrow \infty} \operatorname{dim}\left(\mathcal{H}_{0}^{p} / m_{Y, 0}^{k} \mathcal{H}_{0}^{p}\right) / k^{d},
$$

where $m_{Y, 0}$ denotes the maximal ideal of the local ring $\mathcal{O}_{Y, 0}$. 
By construction the complex $K^{\bullet}\left(f^{-1}-T, \mathcal{O}_{Y, 0}^{E}\right)$ is quasi-isomorphic to the complex

$$
\mathcal{L}^{\bullet}: 0 \rightarrow \mathcal{O}_{Y, 0}^{L^{0}} \stackrel{u^{0}}{\longrightarrow} \mathcal{O}_{Y, 0}^{L^{1}} \stackrel{u^{1}}{\longrightarrow} \cdots \stackrel{u^{n-1}}{\longrightarrow} \mathcal{O}_{Y, 0}^{L^{n}} \rightarrow 0
$$

of finitely generated $\mathcal{O}_{Y, 0}$-modules. Hence we find that

$$
c=d ! \lim _{k \rightarrow \infty} \frac{\operatorname{dim} H^{p}\left(\mathcal{L}^{\bullet}\right) / m_{Y, 0}^{k} H^{p}\left(\mathcal{L}^{\bullet}\right)}{k^{d}} .
$$

Using the additivity of the Samuel multiplicity for short exact sequences of finitely generated $\mathcal{O}_{Y, 0}$-modules, one can show exactly as in the proof of Theorem 2.2 from [5] that

$$
c=d ! \lim _{k \rightarrow \infty} \frac{\operatorname{dim} H^{p}\left(\mathcal{L}^{\bullet} / m_{Y, 0}^{k} \mathcal{L}^{\bullet}\right)}{k^{d}} .
$$

Lemma 2.1. Let $k$ be a positive integer. The cochain map $h=\left(h_{j}\right)_{j=0}^{n}$ induces isomorphisms of cohomology

$$
H^{j}\left(u^{\bullet}, \mathcal{L}^{\bullet} / m_{Y, 0}^{k} \mathcal{L}^{\bullet}\right) \rightarrow H^{j}\left(f^{-1}-T, \mathcal{O}_{Y, 0}^{E} / m_{Y, 0}^{k} \mathcal{O}_{Y, 0}^{E}\right)
$$

for $j=0, \ldots, n$.

Proof. Exactly as in the proof of Lemma 2.1 from [5], the result can be proved by applying standard exactness results for analytically parametrized complexes of Banach spaces (see Lemma 2.1.5 in [5]) to the mapping cone of the cochain map

$$
\mathcal{L}^{\bullet} / m_{Y, 0}^{k} \mathcal{L}^{\bullet} \stackrel{h}{\rightarrow} K^{\bullet}\left(f^{-1}-T, \mathcal{O}_{Y, 0}^{E} / m_{Y, 0}^{k} \mathcal{O}_{Y, 0}^{E}\right) .
$$

To see that all arguments remain correct when the ideal $\left(z_{1}^{k}, \ldots, z_{d}^{k}\right) \subset \mathcal{O}_{Y, 0}$ used in [4] is replaced by the ideal $m_{Y, 0}^{k} \subset \mathcal{O}_{Y, 0}$, the reader should use the identity

$$
m_{Y, 0}^{k} \mathcal{O}(V, E)=\left\{f \in \mathcal{O}(V, E) ; f_{\alpha}=0 \text { for all } \alpha \in \mathbb{N}^{d} \text { with }|\alpha|<k\right\},
$$

where $f_{\alpha}=\left(\partial^{\alpha} f\right)(0)$ and $V$ is an arbitrary open polydisc centred at 0 .

To relate the invariant

$$
c=d ! \lim _{k \rightarrow \infty} \frac{\operatorname{dim} H^{p}\left(f^{-1}-T, \mathcal{O}_{Y, 0}^{E} / m_{Y, 0}^{k} \mathcal{O}_{Y, 0}^{E}\right)}{k^{d}}
$$

to the growth of the cohomology groups $H^{p}\left(T^{k}, E\right)$, we first remark that the isomorphism of local rings

$$
\mathcal{O}_{X, 0} \rightarrow \mathcal{O}_{Y, 0}, \quad(g, U)_{0} \mapsto\left(g \circ f^{-1}, f(U)\right)_{0},
$$

induces isomorphisms of cohomology

$$
H^{j}\left(z-T, \mathcal{O}_{X, 0}^{E} / m_{X, 0}^{k} \mathcal{O}_{X, 0}^{E}\right) \rightarrow H^{j}\left(f^{-1}-T, \mathcal{O}_{Y, 0}^{E} / m_{Y, 0}^{k} \mathcal{O}_{Y, 0}^{E}\right)
$$

for $j=0, \ldots, n$ and $k \geq 1$. We use Proposition 1.1 to replace the ideals $m_{X, 0}^{k}$ by the ideals $\left(z^{k}\right)=\left(z_{1}^{k}, \ldots, z_{n}^{k}\right) \subset \mathcal{O}_{X, 0}$. 
Lemma 2.2. Let $T \in L(E)^{n}$ be a Fredholm tuple as described at the beginning of Section 2, and let the submanifold $X \subset \varrho_{\mathrm{e}}(T)$ be chosen as above. Then, for every integer $k \geq 1$,

$\operatorname{dim} H^{p}\left(z-T, \mathcal{O}_{X, 0}^{E} /\left(z^{k}\right) \mathcal{O}_{X, 0}^{E}\right) \geq \operatorname{dim} H^{p}\left(z-T, \mathcal{O}_{X, 0}^{E} / m_{X, 0}^{k} \mathcal{O}_{X, 0}^{E}\right)$.

Proof. The maximal ideal $m_{X, 0} \subset \mathcal{O}_{X, 0}$ is generated by (the germs of) the functions $f_{1}, \ldots, f_{d}$. Therefore its $k$ th power $m_{X, 0}^{k}$ is generated by the functions $f^{\alpha}$ where $\alpha \in \mathbb{N}^{d}$ runs through all multiindices with $|\alpha|=k$. Let $N_{k, d}$ be the number of those multiindices.

To prove the assertion, we apply Proposition 1.1 to the coherent $\mathcal{O}_{X^{-}}$ sheaves

$$
\begin{gathered}
\mathcal{G}=\mathcal{O}_{X} / \operatorname{Im}\left(\mathcal{O}_{X}^{n} \stackrel{\left(z_{1}^{k}, \ldots, z_{n}^{k}\right)}{\longrightarrow} \mathcal{O}_{X}\right), \\
\mathcal{H}=\mathcal{O}_{X} / \operatorname{Im}\left(\mathcal{O}_{X}^{N_{k, d}} \stackrel{\left(f^{\alpha}\right)_{|\alpha|=k}}{\longrightarrow} \mathcal{O}_{X}\right) .
\end{gathered}
$$

Since $z_{i}^{k} \in m_{X, 0}^{k}$ for $i=1, \ldots, n$ and since the support of both sheaves is the one-point set $\{0\}$, the map

$$
\mathcal{G} \rightarrow \mathcal{H}, \quad\left[(g)_{z}\right] \rightarrow\left[(g)_{z}\right],
$$

is a well-defined epimorphism between coherent $\mathcal{O}_{X}$-sheaves. By Lemma 2.1.5 in [5], applied to the complex $K^{\bullet}\left(f^{-1}(z)-T, E\right)$, it follows that

$$
H^{j}\left(z-T, \mathcal{O}_{X, 0}^{E}\right)=0 \quad(j=p+1, \ldots, n) .
$$

Hence Proposition 1.1 implies that the induced map $H^{p}\left(z-T, \mathcal{G}_{0}^{E}\right) \rightarrow$ $H^{p}\left(z-T, \mathcal{H}_{0}^{E}\right)$ remains surjective. Since the functor of passing from coherent sheaves to the stalks of the sheaf tensorized with $E$ is exact, there are canonical isomorphisms $\mathcal{G}_{0}^{E} \cong \mathcal{O}_{X, 0}^{E} /\left(z^{k}\right) \mathcal{O}_{X, 0}^{E}$ and $\mathcal{H}_{0}^{E} \cong \mathcal{O}_{X, 0}^{E} / m_{X, 0}^{k} \mathcal{O}_{X, 0}^{E}$. This observation completes the proof.

Summarizing the results obtained so far, we obtain the estimate

$$
d ! \liminf _{k \rightarrow \infty} \frac{\operatorname{dim} H^{p}\left(z-T, \mathcal{O}_{X, 0}^{E} /\left(z^{k}\right) \mathcal{O}_{X, 0}^{E}\right)}{k^{d}} \geq c,
$$

where $T$ and $X$ are as described at the beginning of Section 2 and

$$
c=\min \left\{\operatorname{dim} H^{p}(z-T, E) ; z \in X\right\} .
$$

A second application of Proposition 1.1 allows us to replace $\mathcal{O}_{X, 0}^{E}$ by the stalk $\mathcal{O}_{\mathbb{C}^{n}, 0}^{E}$ of all $E$-valued convergent power series near the origin $z=0$ in $\mathbb{C}^{n}$.

Lemma 2.3. Let $T \in L(E)^{n}$ be a Fredholm tuple as described at the beginning of Section 2. With the above notations,

$$
\operatorname{dim} H^{p}\left(z-T, \mathcal{O}_{\mathbb{C}^{n}, 0}^{E} /\left(z^{k}\right) \mathcal{O}_{\mathbb{C}^{n}, 0}^{E}\right) \geq \operatorname{dim} H^{p}\left(z-T, \mathcal{O}_{X, 0}^{E} /\left(z^{k}\right) \mathcal{O}_{X, 0}^{E}\right)
$$

for every integer $k \geq 1$. 
Proof. Let us recall that

$$
A=\left\{z \in \varrho_{\mathrm{e}}(T) ; H^{p}(z-T, E) \neq 0\right\} \subset \varrho_{\mathrm{e}}(T)
$$

is an analytic subset and that $U \subset \mathbb{C}^{n}$ is an open neighbourhood of zero such that $X=A \cap U$ is a $d$-dimensional complex submanifold of $\mathbb{C}^{n}$. Since $X \subset U$ is an analytic subset, the ideal sheaf $\mathcal{I}=i(X)$ of $X$ in $U$, that is, the $\mathcal{O}_{U}$-sheaf given by the presheaf

$$
W \mapsto i(X)(W)=\{g \in \mathcal{O}(W) ; g \mid W \cap X=0\} \quad(W \subset U \text { open }),
$$

is coherent by the theorem of Oka-Cartan (Section 4.2 in [7]).

Let $k \geq 1$ be an integer. Then the sheaves

$$
\mathcal{G}=\mathcal{O}_{U} /\left(z^{k}\right) \mathcal{O}_{U}, \quad \mathcal{H}=\left(\mathcal{O}_{U} / \mathcal{I}\right) /\left(z^{k}\right)\left(\mathcal{O}_{U} / \mathcal{I}\right)
$$

are coherent and the quotient map $\mathcal{O}_{U} \rightarrow \mathcal{O}_{U} / \mathcal{I}$ induces an epimorphism $\mathcal{G} \rightarrow \mathcal{H}$ of coherent $\mathcal{O}_{U}$-sheaves. For each element $a \in\left(\mathcal{O}_{U} / \mathcal{I}\right)_{0}$, there is an analytic function $g_{a} \in \mathcal{O}(W)$ on an open neighbourhood $W$ of 0 in $U$ such that $a=\left(g_{a}\right)_{0}+\mathcal{I}_{0}$, and the map

$$
\left(\mathcal{O}_{U} / \mathcal{I}\right)_{0} \rightarrow \mathcal{O}_{X, 0}, \quad a \mapsto\left(g_{a} \mid W \cap X\right)_{0},
$$

is a well-defined isomorphism of local rings. Since by hypothesis

$$
H^{j}\left(z-T, \mathcal{O}_{X, 0}^{E}\right)=0 \quad(j=p+1, \ldots, n),
$$

Proposition 1.1 implies that the induced map

$$
H^{p}\left(z-T, \mathcal{G}_{0}^{E}\right) \stackrel{q}{\rightarrow} H^{p}\left(z-T, \mathcal{H}_{0}^{E}\right)
$$

is surjective. The observation that there are canonical isomorphisms

$$
\mathcal{G}_{0}^{E} \cong \mathcal{O}_{\mathbb{C}^{n}, 0}^{E} /\left(z^{k}\right) \mathcal{O}_{\mathbb{C}^{n}, 0}^{E}, \quad \mathcal{H}_{0}^{E} \cong \mathcal{O}_{X, 0}^{E} /\left(z^{k}\right) \mathcal{O}_{X, 0}^{E}
$$

completes the proof.

The results obtained so far, together with a base change theorem proved in [4], allow us to prove the first main result of this paper.

TheOrem 2.4. Let $T \in L(E)^{n}$ be a Fredholm tuple such that $H^{p}(T, E)$ $\neq 0$ and $H^{j}(T, E)=0$ for $j=p+1, \ldots, n$. Suppose that 0 is a regular point of the analytic set

$$
A=\left\{z \in \varrho_{\mathrm{e}}(T) ; H^{p}(z-T, E) \neq 0\right\} \subset \varrho_{\mathrm{e}}(T)
$$

and that the dimension $d=\operatorname{dim}_{0}(A)$ of $A$ at $z=0$ is positive. Then, for every connected open neighbourhood $X$ of 0 in $A$ such that $X \subset \mathbb{C}^{n}$ is a submanifold, there exist a proper analytic subset $S \subset X$ and a positive real number $c$ with

$$
\operatorname{dim} H^{p}(z-T, E)>c=\operatorname{dim} H^{p}(w-T, E) \quad(z \in S, w \in X \backslash S) .
$$


The number $c$ is independent of the choice of $X$ and satisfies the estimate

$$
d ! \liminf _{k \rightarrow \infty} \frac{\operatorname{dim} H^{p}\left(T^{k}, E\right)}{k^{d}} \geq c .
$$

Proof. Define $c=\min \left\{\operatorname{dim} H^{p}(z-T, E) ; z \in X\right\}$. By Corollary 9.4.7 in [5], the set

$$
S=\left\{z \in X ; \operatorname{dim} H^{p}(z-T, E)>c\right\}
$$

is a proper analytic subset of $X$. In particular, $S$ has no interior in $X$ (Theorem 9.1.2 in [7]). Let $X_{0}$ be any connected open neighbourhood of 0 in $X$. Define $c_{0}$ and $S_{0}$ as above, but this time with respect to $X_{0}$ instead of $X$. Then $S \cap X_{0}$ and $S_{0}$ are proper analytic subsets of $X_{0}$, and hence Theorem 9.1.2 in [7] implies that $S_{0} \cup\left(S \cap X_{0}\right) \neq X_{0}$. But then it follows that $c=c_{0}$. This argument implies that $c$ is independent of the choice of $X$.

The results obtained before Theorem 2.4 show that

$$
d ! \liminf _{k \rightarrow \infty} \frac{\operatorname{dim} H^{p}\left(z-T, \mathcal{O}_{\mathbb{C}^{n}, 0}^{E} /\left(z^{k}\right) \mathcal{O}_{\mathbb{C}^{n}, 0}^{E}\right)}{k^{d}} \geq c .
$$

Since by Corollary 1.3 in [4] there are vector-space isomorphisms

$$
H^{p}\left(T^{k}, E\right) \cong H^{p}\left(z-T, \mathcal{O}_{\mathbb{C}^{n}, 0}^{E} /\left(z^{k}\right) \mathcal{O}_{\mathbb{C}^{n}, 0}^{E}\right) \quad(k \geq 1),
$$

the proof of Theorem 2.4 is complete.

Suppose that $\operatorname{dim}_{0}(A)=0$, but that $T \in L(E)^{n}$ satisfies all the remaining conditions in Theorem 2.4. Then 0 is an isolated point of $A$, and the only connected open neighbourhood of 0 in $A$ is the set $X=\{0\}$. Choose an open neighbourhood $U$ of 0 in $\mathbb{C}^{n}$ with $U \cap A=\{0\}$ and denote by $\mathcal{I} \subset \mathcal{O}_{U}$ the ideal sheaf of the one-point set $X=\{0\}$ in $U$. Then $\left(z^{k}\right)\left(\mathcal{O}_{U} / \mathcal{I}\right)=\{0\}$ for all integers $k \geq 1$ and, as in the proof of Lemma 2.3, an application of Proposition 1.1 with

$$
\mathcal{G}=\mathcal{O}_{U} /\left(z^{k}\right) \mathcal{O}_{U} \quad \text { and } \quad \mathcal{H}=\mathcal{O}_{U} / \mathcal{I}
$$

leads to the estimates

$$
\operatorname{dim} H^{p}\left(T^{k}, E\right) \geq \operatorname{dim} H^{p}\left(z-T, \mathcal{O}_{X, 0}^{E}\right)=\operatorname{dim} H^{p}(T, E)
$$

for all $k \geq 1$. Thus the assertion of Theorem 2.4 remains true in this case if one defines $c=\operatorname{dim} H^{p}(T, E)$.

Our next aim is to deduce a global version of Theorem 2.4. Fix a commuting tuple $T \in L(E)^{n}$ of bounded operators on a complex Banach space $E$. By Corollary 9.4.7 in [5] the sets

$$
S_{j}=\left\{z \in \varrho_{\mathrm{e}}(T) ; H^{j}(z-T, E) \neq 0\right\} \quad(j=0, \ldots, n)
$$

are analytic subsets of the essential resolvent set $\varrho_{\mathrm{e}}(T)$ of $T$. Hence also the inessential spectrum $\sigma_{\mathrm{F}}(T)=\sigma(T) \backslash \sigma_{\mathrm{e}}(T)=\bigcup_{j=0}^{n} S_{j} \subset \varrho_{\mathrm{e}}(T)$ is an analytic subset. The set $\operatorname{Reg}\left(\sigma_{\mathrm{F}}(T)\right)$ of all regular points of this analytic set is an 
open and dense subset of $\sigma_{\mathrm{F}}(T)$ which forms a complex submanifold of $\mathbb{C}^{n}$ (Section 6.2 in $[7]$ ).

TheOrem 2.5. Let $T \in L(E)^{n}$ be a commuting tuple and let $M$ be a component of $\operatorname{Reg}\left(\sigma_{\mathrm{F}}(T)\right)$ of dimension $d$. Then there is an integer $p \in$ $\{0, \ldots, n\}$ such that

$$
d ! \liminf _{k \rightarrow \infty} \frac{\operatorname{dim} H^{p}\left((z-T)^{k}, E\right)}{k^{d}} \geq \min _{w \in M} \operatorname{dim} H^{p}(w-T, E)>0
$$

for all $z \in M$.

Proof. Let us denote by $h: M \rightarrow[0, \infty]$ the function that assigns to each $z \in M$ the number occurring on the left-hand side of the claimed inequality. Fix $z \in M$. Since $M$ is the union of the analytic subsets $S_{j} \cap M$ $(j=0, \ldots, n)$, the union formula for analytic sets (Section 5.3.1 in [7]) shows that

$$
\max _{j=0, \ldots, n} \operatorname{dim}_{z}\left(S_{j} \cap M\right)=d .
$$

Let $j \in\{0, \ldots, n\}$ with $\operatorname{dim}_{z}\left(S_{j} \cap M\right)=d$. Then $M \subset S_{j}$ by the identity lemma for analytic sets (Lemma 9.1.1 in [7]). Let $p \in\{0, \ldots, n\}$ be maximal with $M \subset S_{p}$.

If $d=0$, then $M=\{z\}$ and $z$ is an isolated point of $\sigma_{\mathrm{F}}(T)$. In this case the assertion follows from the remarks following Theorem 2.4. If $d>0$, then $A=M \cap\left(S_{p+1} \cup \cdots \cup S_{n}\right)$ is a nowhere dense analytic subset of $M$ (Theorem 9.1.2 in [7]). For $z \in M \backslash A$, the claimed estimate follows from Theorem 2.4 applied to $z-T$.

For each fixed integer $k \geq 1$, there is a proper analytic subset $A_{k} \subset M$ such that

$$
\operatorname{dim} H^{p}\left((z-T)^{k}, E\right)=\min _{w \in M} \operatorname{dim} H^{p}\left((w-T)^{k}, E\right)
$$

for all $z \in M \backslash A_{k}$ (Corollary 9.4.7 in [5]). Since proper analytic subsets of the manifold $M$ are zero sets with respect to the surface measure on $M$, it follows that the union of all $A_{k}(k \in \mathbb{N})$ and $A$ is a zero set $Z \subset M$ such that

$$
h(z) \geq h(w) \geq \min _{u \in M} \operatorname{dim} H^{p}(u-T, E)>0
$$

for all $z \in M$ and $w \in M \backslash Z$. This observation completes the proof.

If $T \in L(E)^{n}$ has no isolated inessential spectral points, then $\operatorname{dim}_{z}\left(\sigma_{\mathrm{F}}(T)\right)$ $\geq 1$ for every $z \in \sigma_{\mathrm{F}}(T)$. Using the density of the set $\operatorname{Reg}\left(\sigma_{\mathrm{F}}(T)\right)$ of all smooth points in $\sigma_{\mathrm{F}}(T)$, one obtains the following special case.

Corollary 2.6. Let $T \in L(E)^{n}$ be a commuting tuple and let $d \geq 0$ be a non-negative integer such that $\operatorname{dim} M \geq d$ for every connected component $M$ of $\operatorname{Reg}\left(\sigma_{\mathrm{F}}(T)\right)$. Then the set of all points $z \in \sigma_{\mathrm{F}}(T)$ for which there is 
a $p \in\{0, \ldots, n\}$ with

$$
d ! \liminf _{k \rightarrow \infty} \frac{\operatorname{dim} H^{p}\left((z-T)^{k}, E\right)}{k^{d}} \geq 1
$$

is dense in $\sigma_{\mathrm{F}}(T)$. In particular, if there are no isolated points in $\sigma(T)$ which belong to the essential resolvent set $\varrho_{\mathrm{e}}(T)$, then the above estimate holds true with $d=1$ in a dense set of points $z$ in $\sigma_{\mathrm{F}}(T)$.

Suppose that $z$ is an isolated inessential spectral point of a commuting tuple $T \in L(E)^{n}$. Then by J. L. Taylor's version of Shilov's idempotent theorem (Theorem 4.9 in [8]), there is a direct sum decomposition $E=$ $E_{0} \oplus E_{1}$ of $E$ into closed invariant subspaces $E_{0}, E_{1}$ of $T$ such that

$$
\sigma\left(T \mid E_{0}\right)=\{z\}, \quad \sigma\left(T \mid E_{1}\right)=\sigma(T) \backslash\{z\} .
$$

As $\sigma_{\mathrm{e}}(T)=\sigma_{\mathrm{e}}\left(T \mid E_{0}\right) \cup \sigma_{\mathrm{e}}\left(T \mid E_{1}\right)$, the essential spectrum of $T \mid E_{0}$ is empty. It follows that $\operatorname{dim}\left(E_{0}\right)<\infty$ and

$$
\operatorname{dim} H^{p}\left((z-T)^{k}, E\right)=\operatorname{dim} H^{p}\left(\left(z-T \mid E_{0}\right)^{k}, E_{0}\right) \leq\left(\begin{array}{l}
n \\
p
\end{array}\right) \operatorname{dim}\left(E_{0}\right)
$$

for $p=0, \ldots, n$ and $k \in \mathbb{N}$. Therefore the last part of Corollary 2.6 is no longer true if we drop the condition that $T$ does not have isolated inessential spectral points.

\section{References}

[1] C. Ambrozie and V. Müller, Dominant Taylor spectrum and invariant subspaces, preprint, 2006.

[2] M. Didas, Invariant subpaces for commuting pairs with normal boundary dilation and dominating Taylor spectrum, J. Operator Theory 54 (2005), 169-187.

[3] R. Douglas and K. Yan, Hilbert-Samuel polynomials for Hilbert modules, Indiana Univ. Math. J. 42 (1993), 811-820.

[4] J. Eschmeier, Samuel multiplicity and Fredholm theory, Math. Ann. 339 (2007), 21-35.

[5] J. Eschmeier and M. Putinar, Spectral Decompositions and Analytic Sheaves, London Math. Soc. Monogr. 10, Clarendon Press, Oxford, 1996.

[6] X. Fang, The Fredholm index of a pair of commuting operators, II, preprint, 2006.

[7] H. Grauert and R. Remmert, Coherent Analytic Sheaves, Springer, Berlin, 1984.

[8] J. L. Taylor, The analytic functional calculus for several commuting operators, Acta Math. 125 (1970), 1-48.

Fachrichtung Mathematik

Universität des Saarlandes

Postfach 151150

D-66041 Saarbrücken, Germany

E-mail: eschmei@math.uni-sb.de 